https://doi.org/10.23913/ride.v12i23.1056

Artículos científicos

\title{
Estudio del precio entre la instrucción virtual y la instrucción convencional: estudio de caso en el nivel de instrucción superior
}

Study of the Price between Virtual Instruction and Instruction Conventional: Case Study at the Higher Education Level

Estudo de preços entre a instrução virtual e a instrução convencional: um estudo de caso no nível superior de instrução

Claudio Rafael Vásquez Martínez

Universidad de Guadalajara, México crvasquezm@gmail.com https://orcid.org/0000-0001-6383-270X

Felipe González González Universidad Autónoma de Tamaulipas, México felgonzale@docentes.uat.edu.mx https://orcid.org/0000-0002-1410-8616

Joaquín Torres Mata Universidad Autónoma de Tamaulipas, México jtorresma@docentes.uat.edu.mx https://orcid.org/0000-0002-9298-8831 


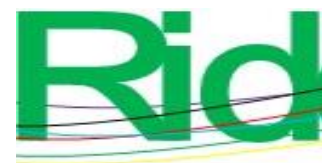

\section{Resumen}

En el presente artículo se realiza un análisis y descripción económica acerca de lo que enfrentan los discentes universitarios de licenciatura y maestría que realizan sus estudios a través de la modalidad de instrucción virtual (IV) en comparación con la instrucción convencional (IC). Asimismo, se detecta el precio semestral y total en esas modalidades. La pregunta formulada fue la siguiente: ¿cuál es el precio total en instrucción virtual (IV) e instrucción convencional (IC), así como el precio total discente-semestre? El estudio se estima para Colombia utilizando información del Instituto Pedagógico. Los resultados indican una notable diferencia entre los precios financieros de acuerdo con los discentes de licenciatura y maestría, en donde para los primeros la instrucción convencional (IC) tiene más precio respecto a la instrucción virtual, mientras que en el caso de los alumnos de maestría, en la instrucción convencional el precio económico resultó menor.

Palabras clave: instrucción convencional, instrucción virtual, precios universitarios.

\section{Abstract}

In this article, an economic analysis and description is carried out about what university undergraduate and master's students face who carry out their studies through the virtual instruction modality (IV) in comparison with conventional instruction (IC). Likewise, the semester and total price is detected in the aforementioned educational modalities. The question asked was the following: what is the total price in virtual instruction (IV) and conventional instruction (IC) as well as the total price for student semester? The study is estimated for Colombia using information from the Pedagogical Institute. The results indicate a notable difference between the financial prices according to undergraduate and master's students, where for the former, conventional instruction (IC) has a higher price compared to virtual instruction, while, in the case of students from master's degree, conventional education the economic cost was less price.

Keywords: conventional instruction, virtual instruction, university prices. 


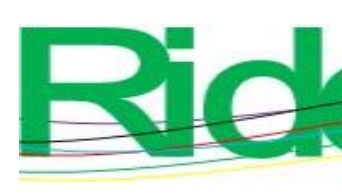

\section{Resumo}

Neste artigo, é realizada uma análise e descrição econômica sobre o que enfrentam os estudantes universitários de bacharelado e mestrado que realizam seus estudos por meio da modalidade de ensino virtual (IV) em comparação com o ensino convencional (IC). Da mesma forma, o preço semestral e total é detectado nessas modalidades. A pergunta feita foi a seguinte: qual é o preço total da instrução virtual (IV) e convencional (IC), assim como o preço total aluno-semestre? O estudo é estimado para a Colômbia a partir de informações do Instituto Pedagógico. Os resultados indicam uma diferença notável entre os preços financeiros segundo alunos de graduação e mestrado, onde para os primeiros o ensino convencional (IC) tem um preço superior ao ensino virtual, enquanto no caso dos alunos de mestrado, no ensino convencional o preço econômico foi diminuir.

Palavras-chave: ensino convencional, ensino virtual, preços universitários.

Fecha Recepción: Abril 2021

Fecha Aceptación: Octubre 2021

\section{Introducción}

La instrucción juega un papel central en la vida social de toda nación, pues con ella se procura el mejoramiento de las habilidades de los ciudadanos para que en un futuro cercano cada persona se incorpore al campo laboral y aporte propuestas que den solución a las necesidades que demanda la sociedad. Si bien la preocupación de algunas instituciones es fomentar el equilibrio - aunque en ese ideal se suelen olvidar las diferencias que pueden presentarse entre los pénsums_-, no es menos cierto que ello se debe en oportunidades a la carencia de instrumentos que permitan identificar la estructura de los precios entre pénsums. Sin embargo, para que lo anterior se vea cristalizado es necesario iniciar y terminar un pénsum educativo en las diferentes disciplinas, ya sea de instrucción convencional (IC) —en un salón de clases - o a través de las diferentes propuestas que engloba la instrucción virtual (IV), las cuales exigen determinados presupuestos a corto, mediano y a largo plazo.

En relación con lo anterior, la formación educativa presencial a nivel internacional se ha convertido en un factor económico productivo que se sostiene en tres columnas: calidad, costos y cobertura. Como señala Daniel (1998), la calidad es una característica directa de los costos y opuesta a la cobertura, de ahí la importancia de su análisis para la provisión de instrucción a los aspirantes a estudios de licenciatura y maestría, grados académicos que podrían verse truncados si se desconocen los precios que cada uno de ellos exige. 

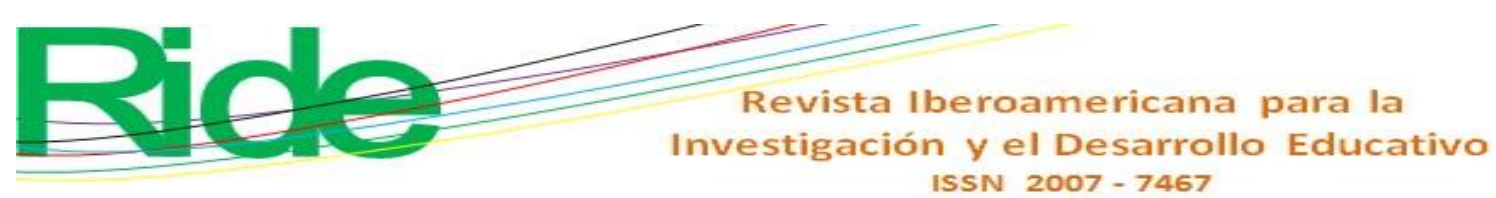

instrucción virtual (IV) son rendimiento de medios, transferencia de contenidos académicos y gerencia (Negi, 2009).

a. Precio: Es el valor producido por el manejo de un pénsum o estructura (Checchi, 2006; John, 2004).

b. Precio por periodo académico: Este concepto hace referencia a la suma de valores aplicables a alguna de las concordancias por alguna denominación en un periodo académico. Incluye enteramente los valores provocados responsablemente de si han sido saldados o no (Jones, 2007; Podmoguilnye, 2006).

c. Precio inmediato: Es el que se adjudica a todos los valores que son atribuibles de primera mano a la instrucción que realizan las distintas correspondencias escolares y que proceden de los procedimientos otorgados expresamente para su cálculo instructivo (Clothier, 2003; Dobson, 2013).

d. Precio crítico: Es aquel producido por los mercados monetarios de las correspondencias gerenciales esenciales, es decir, la gerencia global, utilidad escolar, asistencia de procedimientos, estructura física, imprentas y librerías. Asimismo, todo lo que sirva de apoyo a las correspondencias instruccionales (BenDavid-Hader, 2018; Brimbley, 2002).

Por su parte, Anderson y Raiborn (2007) hacen referencia al concepto precio como el valor que se otorga a los bienes, recursos o servicios adquiridos. Este es el resultado de un sacrificio económico, cuyo valor está determinado por el tipo de cambio monetario. Además, los precios pueden variar según la zona donde se encuentre el discente.

\section{Encuadre instrumental}

Demografía

Se elaboró el empadronamiento con toda la demografía y la información de la relación computarizada suministrada por la secretaría organizativa y la secretaría escolar para las variantes instrucción convencional (IC) e instrucción virtual (IV).

\section{Sinopsis del esquema realizado en la observación de precios totales}

La observación de precios se calculó con referencia fidedigna desde el periodo académico A hasta el I. El precio inmediato se caracteriza por sueldo de los docentes, y los precios críticos están conformados por el precio total de los cargos administrativos (Alexander, 2015). Por ende, los otros precios críticos ocasionados en la pénsum están constituidos por tipo 


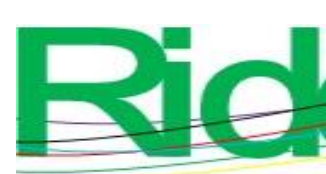

Revista Iberoamericana para la
Investigación y el Desarrollo Educativo
ISSN $2007-7467$

de instrucción convencional (IC) licenciatura, instrucción virtual (IV) licenciatura e instrucción convencional (IC) maestría (Baker, 2008).

$\mathrm{Sj}$ = Adición de los precios inmediatos y los precios críticos de un pénsum académico j.

$\mathrm{k}=$ Subgrafo que distingue el registro de alza por periodo académico.

$\mathrm{Iq}=$ Grafo de alza por periodo académico q admitiendo como variación de precio en relación con el periodo académico A hasta I.

$\mathrm{PT}=$ Precio total del pénsum de instrucción por licenciatura y maestría en moneda constantes en relación con el periodo académico A hasta I.

$$
P T=\left[\sum_{h=1}^{f} S j\right] \cdot\left[\sum_{k=1}^{g} I q\right]
$$

\section{Sobre las prestancias instrumentales}

En el esquema se utiliza la dirección de la información de los precios educativos de los niveles licenciatura y maestría a través de instrumentos en cuya elección se contemplaron comentarios e información de las siguientes secretarías: Planeación Académica, Secretaría Administrativa, Secretaría Académica, Secretaría de Estructura, de Computación, Secretaría de Cuentas, Secretaría de Gastos e Ingresos, Secretaría de Enlace de Empleo y Secretaría de Control Escolar.

Referente al indicador económico, se empleó el grafo de alza de precios por periodo académico de Colombia.

\section{Sobre las restricciones}

Uno de los obstáculos más significativos fue la irregularidad escolar que se ha presentado en el Instituto Pedagógico. En consideración de esta incoherencia observada, se revisó cada periodo académico en forma teórica, es decir, que al resolver los precios relativos por periodo académico se distribuyó de la conjetura que se había avanzado regularmente sin contratiempos. Una vez alcanzados los precios inmediatos y críticos, teóricos por periodo académico, se aclararon considerando la irregularidad escolar. 


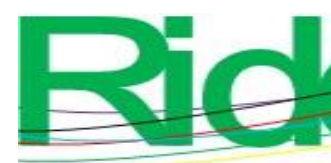

\section{Revista Iberoamericana para la Investigación y el Desarrollo Educativo ISSN $2007-7467$}

Centrándose solo en el precio real, la instrucción convencional (IC) maestría alcanzó un precio discente-asignatura más elevado que los otros tipos instruccionales, con \$2,012 moneda corriente.

Diferenciando este mismo precio en el ámbito de licenciatura, la instrucción convencional (IC) indicó un precio inferior a la instrucción virtual (IV), equivalente a \$ 305.00 y \$ 894.00 monedas corrientes, respectivamente (tabla 2).

Tabla 2. Cambio histórico del precio discente-asignatura por periodo académico en el pénsum de instrucción, Instituto Pedagógico en monedas corrientes

\begin{tabular}{|l|l|l|l|l|l|l|l|l|l|}
\hline $\begin{array}{l}\text { Periodo } \\
\text { académico } \\
\begin{array}{l}\text { Pénsum } \\
\text { de instrucción }\end{array}\end{array}$ & A & B & C & D & E & F & G & H & I \\
\hline Total general & 56 & 48 & 80 & 114 & 120 & 111 & 118 & 143 & 157 \\
\hline EC maestría & 1153 & 1877 & 1392 & 1461 & 1356 & 1804 & 1554 & 1853 & 2012 \\
\hline EC licenciatura & 64 & 95 & 171 & 182 & 197 & 2016 & 251 & 278 & 305 \\
\hline ENP licenciatura & 288 & 2316 & 383 & 602 & 632 & 569 & 578 & 788 & 894 \\
\hline
\end{tabular}

Fuente: Elaboración propia

Enmarcado en este periodo académico, el precio real fue de \$ 25,575.00 moneda corriente (tabla 3 ).

Tabla 3. Cambio histórico del precio total discente-periodo académico en el pénsum de instrucción, Instituto Pedagógico

\begin{tabular}{|l|l|l|l|l|l|l|l|l|l|}
\hline $\begin{array}{l}\text { Periodo } \\
\text { académico } \\
\begin{array}{l}\text { Pénsum } \\
\text { de instrucción }\end{array}\end{array}$ & A & B & C & D & E & F & G & H & I \\
\hline Total general & 25575 & 20564 & 41194 & 60236 & 64081 & 63773 & 67513 & 82105 & 90518 \\
\hline EC maestría & 51810 & 44978 & 59778 & 62722 & 56882 & 72066 & 73962 & 81515 & 89840 \\
\hline EC licenciatura & 25857 & 38626 & 61922 & 83634 & 96002 & 108162 & 129614 & 146929 & 164244 \\
\hline ENP licenciatura & 8914 & 9250 & 10290 & 19826 & 29578 & 20370 & 31703 & 39740 & 50055 \\
\hline
\end{tabular}

Fuente: Elaboración propia 


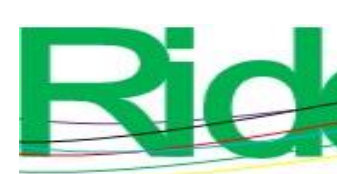

Por consiguiente, el precio significó $24.28 \%$ del referido en el periodo académico A, disparando una repercusión baja de gradiente de $2.26 \%$ en moneda constante. Al considerar los guarismos de gradiente, del precio discente-periodo académico a precios corrientes, se atisba que si bien han incrementado, el transcurso encarecedor ha aminorado su repercusión, al instante de manifestar repercusiones bajas en algunos de ellos (Clothier, 2003; Ugeux, 2014).

\section{Discusión}

El tema de educación presencial o a distancia en términos de su precio, así como la definición de su concepto en sí mismo es — hasta la fecha, y sobre todo en esta época tecnológica - un tópico que da para variadas discusiones. Si a lo anterior agregamos la necesidad de evaluar las diferentes modalidades de instrucción, la tarea se convierte en una nueva labor para quien intenta conceptualizar y definir la manera de llevar a cabo el proceso de enseñanza-aprendizaje.

En tal sentido, el porcentaje de adición de los precios inmediatos y los precios críticos de un pénsum académico propuesto aquí no debe confundirse con el índice de anclado en una fecha específica en la que se realizó este estudio científico, puesto que se refiere a cifras que pueden variar con el tiempo. Por eso, los porcentajes del sobreprecio de la educación presencial podrían cambiar hasta el punto de ser más económica, siempre que se cumplan los compromisos y funciones de toda universidad, es decir, transmisión de la información, cobertura, equidad y generación del conocimiento (Maza-Ávila, Vergara-Schmalbach y Romero, 2017).

Además, hay que tener en cuenta que para comparar el monetario entre dos modalidades educativas no solo deben pertenecer a una misma institución, sino que además se debe tener la seguridad de que las carreras de licenciatura y posgrado analizadas sean de un mismo ciclo escolar.

Al expresar y valorar los efectos alcanzados, la instrucción virtual (IV) es factible económicamente en menor grado, siempre y cuando las opciones que se apliquen estén de acuerdo con la medida de lo poblado que estudian y sean apropiados desde el parámetro de los precios (Michael, 2005). En su momento, en la instrucción virtual (IV) existe más obligación a las variantes realizadas por modificaciones en las habilidades organizacionales (Ugeux, 2014). Esto involucra que la aceptación económica de los ejemplos en instrucción virtual (IV) reducidos se derivan en mucho del razonamiento que se aplique en el indicativo de políticas y de una gestión ágil (Thompson et al., 2000). 


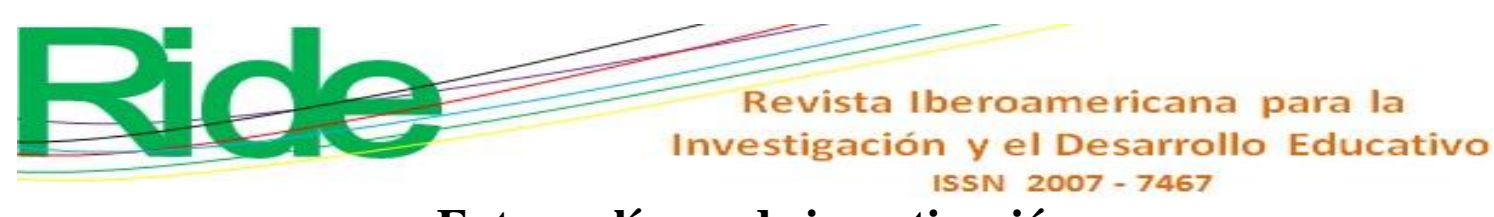

Futuras líneas de investigación

De este estudio se desprenden las siguientes futuras líneas de investigación:

- Indagaciones en aulas de clase de primaria, secundaria, preparatoria, licenciatura y maestría sobre los precios que realizan.

- Estudios locales en instituciones educativas sobre los efectos de las pandemias y el periodo de pandemias en los precios que realizan en sus integrantes.

\section{Referencias}

Alexander, K. (2015). Financing public schools: theory, policy and practice. New York: Routlege.

Anderson, H. R. y Raiborn, M. H. (2007). Conceptos básicos de contabilidad de precios. México. D.F. Grupo Editorial Patria.

Aubry, C. (2015). Education and the state: international perspective on a changing relationship. New York: Routlege.

Baker, B. P. (2008). Financing education systems. Upper Saddle River, N.J.: Pearson/Merrill/PrenticeHall.

BenDavid-Hadar, I. (ed.) (2018). Education Finance, Equality and Equity. Cham. Springer International Publishing.

Baum, S. (2016). Student debt: rhetoric and realities of higher education financing. New York: Palgrave Macmillan.

Brimley, V. (2002). Financing education in a climate of charge. Boston: Allym and Bacon.

Canning, M. (2007). Higher education financing in the new EU member states: leveling the playing field. Washington, D.C.: World Bank.

Chapman, B. (2014). Income contingent loans; theory, practice and prospects. New York: Palgrave Macmillan.

Checchi, D. (2006). Economics of education: human capital, family background and inequality. Cambridge: Cambridge University Press.

Clothier, S. (2003). Financing early care and education: funding and policy choices in a changing fiscal environment. Denver: National Conference of State Legislatures.

Daniel, J. (1998). Mega-universities \& knowledge media. Technology strategies for higher education. London: Kogan Page.

Dobson, W. (2013). Human capital formation and economic growth in Asia and the Pacific. New York: Routledge, Taylor and Francis Group. 


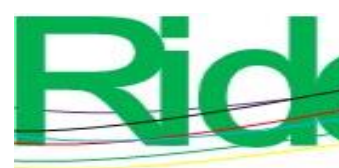

Revista Iberoamericana para la
Investigación y el Desarrollo Educativo
ISSN $2007-7467$

Duart, J. M. (2000). Aprender sin distancias. Barcelona: Universitat Oberta de Catalunya. Recuperado de http://www.uoc.edu/web/esp/articles/josep _ maria _ duart.html

Fabian, M. (2018). Hybrid public policy innovations: contemporary policy beyond ideology.

New York: Routledge, Taylor \& Francis Group.

Gerard, M. (2014). Mobility of students and the highly skilled: implications for education financing and economic policy. Cambridge, MA: MIT Pres.

John, E. (2004). Public Funding of higher education: changing contenxts and new rationales. Baltimore: Johns Hopkins Univesity Press.

Jones, P. W. (2007). World Bank Financing of education: lending, learning and development. New York: Routledge, Taylor \& Francis Group.

Johnstone, D. B. (2010). Financing higher education worldwide who pays? Who should pay? Batimore: Johns Hopkins University Press.

Maza-Ávila, F. J., Vergara-Schmalbach, J. C. y Romero, R. R. (2017). Eficiencia y productividad en la cobertura de las universidades públicas colombianas. Investigación \& Desarrollo, 25(2), 6-33. Recuperado de https://www.redalyc.org/articulo.oa?id=26854666001

Michael, S. D. (2005). Financing higher education in a global market. New York: Algora Pub. Negi, U. R. (2009). Financing higher education. New Delhi: Association of Indian Universities. Podmoguilnye, M. G. (2006). El costeo basado en actividades. Buenos Aires: La Ley.

Patti, R. J. (2009). Handbook of human services management. Los Angeles: SAGE.

Ripoll, V. y Balada T. (2003). Manual de precios. Barcelona: Gestión 2000.

Rodríguez S. (2012). El modelo CANOA/Universidades: características generales. Personalización e implantación: problemas prácticos y resumen de la situación actual. Jornada sobre implantación de la contabilidad analítica en las universidades españolas. Valencia: Universidad de Valencia.

Seligman, A. I. (2012). Is graduate schol really for you?: the whos, whats, hows and whys of pursuing a masters or PhD. Baltimore: Johns Hopkins University Press.

Thompson, A. A., Peteraf, M., Gamble, J. y Strickland, A. J. (2000). Administración estratégica. Teoría y casos. México: McGraw-Hill.

Unesco (2003). Financing education: investments and returns: analysis of the world education indicators. Paris: UNESCO Institute for statistics.

Ugeux, G. (2014). International Finance regulation: the quest for Financial stability. Hoboken: Wiley. 


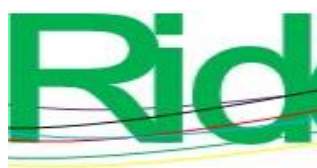

Revista Iberoamericana para la Investigación y el Desarrollo Educativo ISSN $2007-7467$

Zumeta, W. (2012). Financing American higher education in the era of globalization.

Cambridge, Massachusetts: Harvard Education Press.

\begin{tabular}{|c|c|}
\hline Rol de Contribución & Autor (es) \\
\hline Conceptualización & $\begin{array}{l}\text { Claudio-Rafael Vásquez-Martínez, igual; Felipe González } \\
\text { González, igual; Joaquín Torres Mata, igual. }\end{array}$ \\
\hline Metodología & $\begin{array}{l}\text { Claudio-Rafael Vásquez-Martínez, igual; Felipe González } \\
\text { González, igual; Joaquín Torres Mata, igual. }\end{array}$ \\
\hline Software & $\begin{array}{l}\text { Claudio-Rafael Vásquez-Martínez, igual; Felipe González } \\
\text { González, igual; Joaquín Torres Mata, igual. }\end{array}$ \\
\hline Validación & $\begin{array}{l}\text { Claudio-Rafael Vásquez-Martínez, igual; Felipe González } \\
\text { González, igual; Joaquín Torres Mata, igual. }\end{array}$ \\
\hline Análisis Formal & $\begin{array}{l}\text { Claudio-Rafael Vásquez-Martínez, igual; Felipe González } \\
\text { González, igual; Joaquín Torres Mata, igual. }\end{array}$ \\
\hline Investigación & $\begin{array}{l}\text { Claudio-Rafael Vásquez-Martínez, igual; Felipe González } \\
\text { González, igual; Joaquín Torres Mata, igual. }\end{array}$ \\
\hline Recursos & $\begin{array}{l}\text { Claudio-Rafael Vásquez-Martínez, igual; Felipe González } \\
\text { González, igual; Joaquín Torres Mata, igual. }\end{array}$ \\
\hline Curación de datos & $\begin{array}{l}\text { Claudio-Rafael Vásquez-Martínez, igual; Felipe González } \\
\text { González, igual; Joaquín Torres Mata, igual. }\end{array}$ \\
\hline $\begin{array}{l}\text { Escritura - Preparación del } \\
\text { borrador original }\end{array}$ & $\begin{array}{l}\text { Claudio-Rafael Vásquez-Martínez, igual; Felipe González } \\
\text { González, igual; Joaquín Torres Mata, igual. }\end{array}$ \\
\hline $\begin{array}{l}\text { Escritura - Revisión y } \\
\text { edición }\end{array}$ & $\begin{array}{l}\text { Claudio-Rafael Vásquez-Martínez, igual; Felipe González } \\
\text { González, igual; Joaquín Torres Mata, igual. }\end{array}$ \\
\hline Visualización & $\begin{array}{l}\text { Claudio-Rafael Vásquez-Martínez, igual; Felipe González } \\
\text { González, igual; Joaquín Torres Mata, igual. }\end{array}$ \\
\hline Supervisión & $\begin{array}{l}\text { Claudio-Rafael Vásquez-Martínez, igual; Felipe González } \\
\text { González, igual; Joaquín Torres Mata, igual. }\end{array}$ \\
\hline Administración de Proyectos & $\begin{array}{l}\text { Claudio-Rafael Vásquez-Martínez, igual; Felipe González } \\
\text { González, igual; Joaquín Torres Mata, igual. }\end{array}$ \\
\hline Adquisición de fondos & $\begin{array}{l}\text { Claudio-Rafael Vásquez-Martínez, igual; Felipe González } \\
\text { González, igual; Joaquín Torres Mata, igual. }\end{array}$ \\
\hline
\end{tabular}

